\title{
Técnicas de inteligencia artificial aplicadas al análisis de imágenes diagnóstico
}

\author{
Artificial intelligence techniques applied to the analysis of diagnostic \\ images
}

\author{
Adriana Milena Machacado-Rojas ${ }^{\mathrm{a}^{*}}$, Lilia Edith Aparicio-Pico ${ }^{\mathrm{b}}$ \\ a*Ingeniería Electrónica, ammachacador@correo.udistrital.edu.co, https://orcid.org/0000-0001-5140-6420, universidad Distrital \\ Francisco José de Caldas, Bogotá D.C., Colombia \\ bDoctora en Ciencias Técnicas, medicina@udistrital.edu.co, https://orcid.org/0000-0003-1841-4423, universidad Distrital Francisco \\ José de Caldas, Bogotá D.C., Colombia
}

Forma de citar: Machacado Rojas, A. M., \& Aparicio Pico, L. E. (2021). Técnicas de inteligencia artificial aplicadas al análisis de imágenes diagnóstico. Eco Matemático, 12 (2), 100-111

Recibido: $15 / 04 / 2021$

Aceptado: 18/5/2021

\section{Palabras clave \\ Inteligencia artificial, redes neuronales, imágenes, automático, diagnóstico, técnicas, precisión.}

Resumen:La implementación de la Inteligencia Artificial (IA) en los procedimientos médicos ha contribuido a optimizar la prevención y el seguimiento de algunos tratamientos médicos. Esta tecnología de vanguardia es ampliamente utilizada en el procesamiento de imágenes médicas debido a su eficiencia para revelar enfermedades o cuerpos extraños en un tiempo más corto.

El presente artículo revisa algunas características, después de una recopilación de información, sobre el uso de tecnologías de Inteligencia Artificial para el diagnóstico de enfermedades por imágenes. Para cumplir con esto, fue necesario indagar sobre algunos tipos de Diagnóstico por Imagen (DI) como tomografía computarizada, ultrasonido, resonancia magnética y radiología. La investigación arrojó que el primer tipo de DI es el más utilizado y conocido por los centros de salud y laboratorios que brindan este tipo de servicio en Colombia. Esto puede deberse a múltiples factores, principalmente a su amplia disponibilidad, su fácil funcionamiento, su escaso uso de radiación y su bajo costo. De hecho, su aprobación como método en la detección de diversas enfermedades es tan simple que no requiere más trámites administrativos.

Por lo tanto, esta revisión pretende presentar brevemente al lector la información técnica en cuanto a imágenes médicas. Primero, presentando algunos métodos y funciones. En segundo lugar, mostrando los avances más recientes en este campo de estudio y su contribución a la mitigación del problema de salud pública más reciente llamado nuevo coronavirus.

*Autor para correspondencia: ammachacador@correo.udistrital.edu.co

https://doi.org/10.22463/17948231.3237

2462-8794(C) 2021 Universidad Francisco de Paula Santander. Este es un artículo bajo la licencia CC BY 4.0 


\section{Keywords:}

Artificial Intelligence, neural networks, images, logic, automatic, diagnosis, techniques, precision.

\section{Introducción}

Las imágenes representan en el ámbito de la medicina una herramienta que determina con mayor claridad y exactitud el diagnóstico de patologías o enfermedades en los pacientes, esto en complementación con otros tipos de exámenes; de otra parte. El DI ha estado en constante desarrollo e investigación para la optimización y eficiencia en la toma de datos y análisis de los mismos. Uno de los principales alcances ha sido asociarle técnicas de procesamiento para obtener mayor eficiencia en el diagnóstico de enfermedades a base del análisis de imágenes.

Existe un conjunto sólido de razones suficientes para comprender el trascendente papel de las computadoras, específicamente de la Inteligencia Artificial en la sociedad postmoderna, de las Tecnologías de la información y las Comunicaciones TICs en general y otras tecnologías asociadas.
La implementación de Inteligencia Artificial en procedimientos de la medicina es válida actualmente, porque implica optimización en la medicina preventiva como en el seguimiento del tratamiento, además, los procedimientos que constituyen la toma de imágenes como punto esencial para el diagnóstico a tiempo de muchas patologías o posibles enfermedades facilitan la realización de un juicio médico rápido y eficiente y el desarrollo de tecnologías asociadas con las técnicas de IA, aplicadas a la medicina, representa una novedosa perspectiva, que puede reducir los costos, el tiempo, los errores médicos; así como potenciar el uso de los recursos humanos en las ramas médicas con mayores requerimientos (Expósito Gallardo \& Ávila Ávila, 2008).

Desde el descubrimiento del área de estudio de IA, la ciencia ha estado en constante desarrollo para la implantación de métodos y técnicas de éste campo, que facilitan procedimientos ya existentes, 
dentro del ciencias médicas, económicas, sociales y otras.

Este artículo recopila gran parte de los últimos avances en la automatización con técnicas y métodos de la IA en el análisis de patologías por medio de imágenes diagnósticos, surgiendo las siguientes preguntas de investigación.

Q1: ¿Cómo se determina la automatización en imágenes diagnóstico con IA?

Q2: ¿Cuál ha sido los avances en proyectos de investigación sobre DI en la medida de la precisión para determinar la viabilidad del método implementado?

Q3: ¿Cuáles son limitaciones presentadas en las propuestas estudiadas y los alcances o posibles proyectos a realizar a partir de estos estudios de técnicas de IA en imágenes diagnóstico?

Q4: ¿Qué fundamentos matemáticos aplica gran parte de las tecnologías de IA en análisis de imagen?

Q5: ¿Qué avances y proyectos han implementado tecnologías de IA para una detección rápida y segura de la enfermedad de Covid-19 desde una imagen médica?

La recopilación y selección de estudios e información sobre avances de implantación de Técnicas de IAen el campo de imágenes diagnósticos, permite tener una visión actualizada de este tema y sirve como punto de partida para futuros trabajos $\mathrm{y}$ proyectos que tienen como objetivo el progreso en la determinación de enfermedades a base de imágenes médicas.

Teniendo en cuenta los anteriores datos e información es pertinente que surja la siguiente pregunta en la conclusión:

Q6: ¿Un artículo científico de Revisión sobre los avances y estudios de las Técnicas de IA aplicadas al análisis de imágenes diagnóstico, facilitará la realización y conocimientos de estos procedimientos a la comunidad científica y académica?

\section{Método}

El método implementado para éste artículo en la recopilación y organización de información, es el de revisión sistemática según las directrices PRISMA. Este plantea las etapas de planeación, selección, extracción y ejecución.

Técnicas de IA en imágenes

Diagnóstico

Sintaxis de búsqueda: con operadores AND y OR.

Filtro de últmos

10 años.

Elaboración

EJECUCIÓN

Figura 1. Proceso de revisión implementado 


\section{A. Planeación}

En esta etapa se identifica el problema a abordar en el artículo y la formulación de la pregunta objetivo del articulo presente. Se selecciona las fuentes principales de búsqueda de base de datos generales como google scholar y BD UDFJC (Base de datos de la universidad Distrital Francisco José de Caldas) y fuentes específicas como ScienceDirect, PubMed e IEEE Digital Library.

\section{B. Selección}

Dentro de los estudios por imágenes se encuentra: rayos X, Tomografía computarizada (TC), ultrasonido y resonancia Magnética (MRI); siendo los rayos X y la tomografía computarizada los tipos de imágenes con mayor avance y estudio en el uso de técnicas de inteligencia artificial. Para la realización de búsqueda de información en las diferentes fuentes o base de datos se usó criterios de búsqueda determinados.

Tabla I. Sintaxis implementada en la búsqueda de información

\section{Criterios de búsqueda}

(Artificial Intelligence techniques for diagnostic image analysis) OR (AI techniques in diagnostic images) ((( AI techniques for X-ray analysis) OR (x-ray analysis with AI techniques)) AND ((AI techniques for TC analysis) OR (TC analysis with AI techniques))) AND ((AI techniques for ultrasound analysis) OR (ultrasound analysis with AI techniques))) AND ((AI techniques for analysis MRI) OR (MRI analysis with AI techniques))

Fuente: Autor

Ingresando el criterio de búsqueda de la Tabla I. en el buscador google scholar, se obtiene un total de 4660 resultados, después para disminuir este número teniendo en cuenta el alcance este este artículo, se aplica filtros y opciones del buscador. El primer filtro es el rango de publicación desde 2011 hasta el 2021, para obtener 3290 resultados. Por último, se ordena los artículos por relevancia.

\section{Extracción}

En esta sesión se realiza la gestión y depuración de la información, se estudia los avances del tema desde el 2011 hasta el 2021. De la información obtenida y ordenada, se selecciona los primeros 100 artículos teniendo en cuenta la lectura del título, que comprenda específicamente el uso de técnicas de IA en algún tipo de DI.

Se realiza una selección y clasificación de la información investigada para exponerla en el presente artículo según sesiones o áreas de interés. Se evalúa la calidad de los estudios para la estructuración del presente artículo con la previa lectura del abstrac o resumen, para un nuevo grupo de selección de 60 estudios.

\section{Ejecución}

En la presente fase se procede a estudiar cada artículo o información del grupo final seleccionado. Se resume aspectos importantes en alcances y limitaciones. Posteriormente se selecciona un total de 23 estudios, en este último grupo de selección se tiene en cuenta abarcar avances en los diferentes tipos de imágenes diagnósticos, los criterios de patologías o enfermedades de mayores riegos, presentación de futuros alcances y principalmente estudios que presentaron resultados estadísticos específicos de precisión en la metodología utilizada.

\section{Resultados y Discusión}

En este punto se expondrá los estudios investigados que propusieron y marcaron un avance tecnológico en el tema principal del artículo. 
Se Agrupa todos los artículos estudiados según temas de interés para el presente desarrollo sobre análisis de imágenes diagnóstico implementando tecnologías de IA, dando respuesta a las preguntas de investigación formuladas inicialmente

\section{A. Niveles de Automatización de IA en imágenes diagnóstico}

En el artículo (Nakata, 2019), el autor propuso los niveles de automatización de la Inteligencia artificial en radiología diagnóstica desde el nivel 0 hasta el nivel 4.

La clasificación de los niveles de automatización es un indicador para la evaluación de los procesos de investigación y desarrollo. (Nakata, 2019).

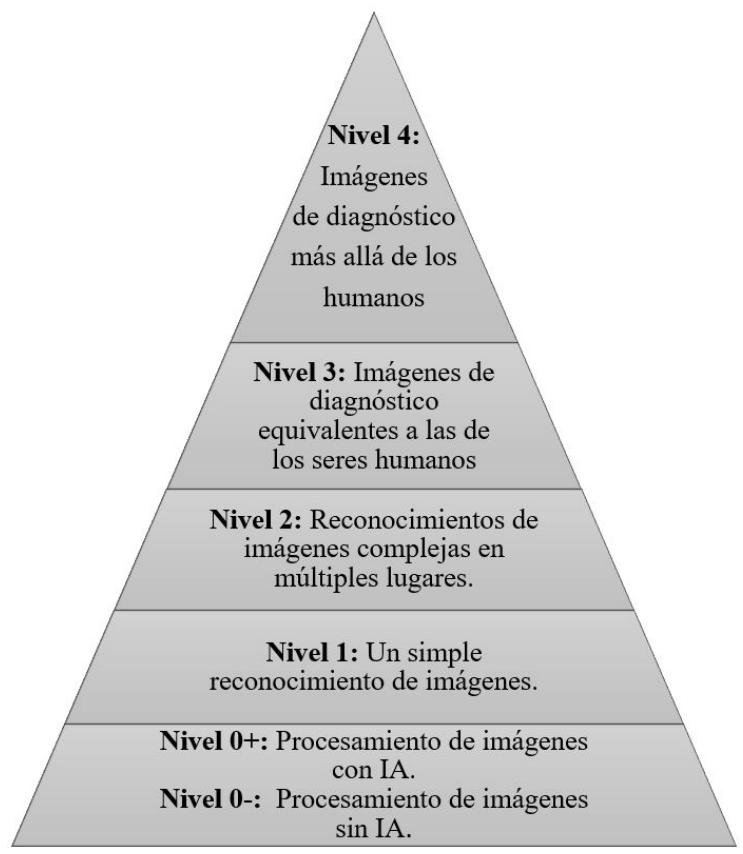

Figura 2. Niveles de automatización de IA de DI según (Nakata, 2019)

Fuente: Autor

En la clasificación de los niveles de automatización de imágenes diagnóstico, el nivel 0 se subdivide en $0+$ y 0 -, donde el primero corresponde al procesamiento de imágenes con Inteligencia artificial, recientemente las nuevas investigaciones de imágenes sintéticas que utilizan GAN (Redes generativas antagónicas) han progresado rápidamente y la segunda subdivisión corresponde al procesamiento de imágenes sin Inteligencia artificial. El nivel 1 es el diagnóstico asistido por computadora de un solo tipo de reconocimiento de imágenes, como la detección de nódulos pulmonares en la TC de tórax. El nivel 2 es el reconocimiento de imágenes complejas en múltiples lugares, como nódulos pulmonares, lesiones de neumonía y lesiones de masas hepáticas. El nivel 3 tiene capacidades de diagnóstico por imagen equivalentes a las de los humanos. El nivel 4 es la capacidad de diagnóstico por imágenes más allá de los humanos (Nakata, 2019).

La determinación de los niveles presentados en la Figura 2. Permite una visión en la clasificación de la información investigada para exponerla según sesiones o áreas de interés.

\section{B. Propuestas o proyectos sobre el diagnóstico de imágenes clínicas implementando IA}

En la búsqueda y recopilación de información en fuentes científicas, se encuentra en común la finalidad de mejorar el procedimiento de análisis de una imagen clínica realizada para determinar diferentes patologías presentadas en diferentes grupos de población. Desde patologías o enfermedades en grupos específicos como adultos de cierto rango de edad o en general y en niños.

Se supera limitaciones presentadas en métodos tradicionales por visión de un profesional, permitiendo estudiar con mayor detalle una imagen en su totalidad, esto evita la exposición recurrente o repetición de procedimientos de toma de imágenes diagnóstico por errores de análisis del profesional o la tecnología tradicional.

Se estudia ese valor especifico de precisión con procedimientos de toma, selección y análisis de imágenes médicas con la implementación de algún 
método o técnica de IA, mejorando la realización y resultados de un tratamiento médico desde un diagnóstico verídico, todo esto sumado con la habilidad y conocimientos del profesional que realiza cada procedimiento.

Tabla II. Estudios relacionados con la aplicación de técnicas de IA en DI

\begin{tabular}{|c|c|c|c|c|}
\hline Literatura & Modalidad & Aplicación & Método/Técnica & Precisión \\
\hline (Fos Guarinos, 2016) & $\mathrm{RX}$ & Radiografias de tórax. & CNN AlexNet. & $87 \%$ \\
\hline (Iturrioz et al., 2018) & TC & Porcentaje de enfisema. & $\begin{array}{l}\text { Cuantificación basado en una } \\
\text { CNN. }\end{array}$ & $90,73 \%$ \\
\hline (Lee et al., 2020) & $\mathrm{RX}$ & Fractura de fémur. & GoogLeNet y Bi-LSTM. & $86,78 \%$ \\
\hline (Chen et al., 2020) & $\mathrm{RX}$ & Radiografia de tórax. & $\begin{array}{l}\mathrm{CNN} \text { : DenseNet o ResNet y } \\
\text { arquitectura YOLOv3. }\end{array}$ & $92,47 \%$ \\
\hline (Nguyen et al., 2020) & US & $\begin{array}{l}\text { Nódulos } \\
\text { malignos. }\end{array} \quad$ tiroideos & CNN ResNet50. & $82.412 \%$ \\
\hline (Tandel et al., 2020) & RM & $\begin{array}{l}\text { Clasificación de tumores } \\
\text { cerebrales. }\end{array}$ & CNN, AlexNet. & $99,20 \%$ \\
\hline (Battineni et al., 2020) & RM & $\begin{array}{ll}\text { Enfermedad } & \text { de } \\
\text { Alzheimer (EA) }\end{array}$ & $\begin{array}{l}\text { Conjunto o híbrido de cuatro } \\
\text { modelos predictivos NB, } \\
\text { ANN,KNN y SVM. }\end{array}$ & $96,12 \%$ \\
\hline $\begin{array}{l}\text { (Vasconcelos et al., 2020) } \\
\text { (Sarhan, 2020) }\end{array}$ & $\begin{array}{l}\text { TC } \\
\text { RM }\end{array}$ & $\begin{array}{l}\text { TC del cerebro. } \\
\text { Clasificación de tumores } \\
\text { cerebrales }\end{array}$ & $\begin{array}{l}\text { Adaptive ABTD. } \\
\text { CAD, (SVM). }\end{array}$ & $\begin{array}{l}98.13 \% \\
99,3 \%\end{array}$ \\
\hline (Faleiros et al., 2020) & $\mathrm{RM}$ & Sacroileítis inflamatoria. & $\begin{array}{l}\text { SVM, perceptrón multicapa } \\
\text { (MLP), el algoritmo basado en } \\
\text { instancias. }\end{array}$ & $95,6 \%$ \\
\hline (Grist et al., 2020) & $\mathrm{RM}$ & $\begin{array}{l}\text { Tumores } \\
\text { pediátricos. }\end{array}$ & AdaBoost. & $85 \%$ \\
\hline (Huang et al., 2020) & RM & Tumores cerebrales. & DFM. & $98 \%$ \\
\hline (Zhang et al., 2020) & US & $\begin{array}{l}\text { Detección auxiliar de } \\
\text { lesiones mamarias. }\end{array}$ & Lightweight neural network. & $91 \%$ \\
\hline (Skandha et al., 2020) & US & Ecoorafia carotídea. & $\begin{array}{l}\text { Deep Convolutional Neural } \\
\text { Network (DCNN) }\end{array}$ & $95,66 \%$, \\
\hline & & & $\begin{array}{l}\text { Visual Geometric Group-16 } \\
\text { (VGG16). }\end{array}$ & \\
\hline (Wang et al., 2020) & RM & $\begin{array}{l}\text { Resección circunferencial } \\
\text { para cáncer de recto. }\end{array}$ & Faster R-CNN M. & $93,2 \%$ \\
\hline
\end{tabular}

Fuente: Autor

En la Tabla II, se expone le precisión lograda en cada propuesta de investigación estudiada. Teniendo en cuenta el ámbito médico en el que se centra cada referencia para el diagnóstico de diferentes enfermedades o patologías, este cálculo utiliza una métrica de exactitud estándar con la siguiente ecuación,

$$
\text { Accuracy }=\frac{T P+T N}{T P+T N+F P+F N}
$$

Donde en la ecuación 1. TP es verdadero positivo, TN es verdadero negativo, $\mathrm{FP}$ es falso positivo y FN es falso negativo (Bekhet et al., 2020).
Con esta métrica de comprobación se proporciona un dato sobre la viabilidad y rendimiento de cada propuesta de investigación dentro del grupo de algoritmos, técnicas o métodos de IA para el DI. Esta medida proporciona información en escala de rendimiento del modelo aplicado en comparación a prácticas tradicionales. 
Tabla III. Tecnología de IA en DI

\begin{tabular}{ll}
\hline \multicolumn{1}{c}{ Técnica } & \multicolumn{1}{c}{ Apuntes sobre la Metodología IA. } \\
\hline AlexNet & $\begin{array}{l}\text { Preentrenada con ImageNet en MATLAB para extraer características de las imágenes y luego usarlas para } \\
\text { entrenar a un clasificador binario (Fos Guarinos, 2016). }\end{array}$ \\
GoogLeNet & $\begin{array}{l}\text { Red neuronal estructurada codificador-decodificador que utiliza informes de radiología como información } \\
\text { auxiliar en el entrenamiento(Lee et al., 2020). }\end{array}$ \\
ResNet & Modelo de aprendizaje por transferencia basado en una red residual (Chen et al., 2020). \\
& $\begin{array}{l}\text { A la salida de cada red se podría obtener una puntuación de clasificación que presenta la probabilidad de } \\
\text { que la imagen de entrada pertenezca a la clase benigna o maligna (Nguyen et al., 2020). }\end{array}$
\end{tabular}

$\begin{array}{ll}\text { SVM } & \begin{array}{l}\text { Son algoritmos de aprendizaje supervisado que se han implementado ampliamente en aplicaciones de } \\ \text { clasificación. Aplicación con descomposición Wavelet, sin realizar ninguna segmentación en la imagen de } \\ \text { entrada (Sarhan, 2020). }\end{array} \\ \text { MLP } & \begin{array}{l}\text { Si la escalabilidad es importante, puede ser la mejor opción de clasificador usando el método Wrapper. } \\ \text { (Faleiros et al., 2020). }\end{array} \\ \begin{array}{l}\text { Adaptive } \\ \text { ABTD }\end{array} & \begin{array}{l}\text { El método propuesto analiza la imagen píxel por píxel y verifica la vecindad del píxel para decidir a qué } \\ \text { región de denidad pertenece ese píxel. Este enfoque crea un nuevo mapa a partir del cual se puede }\end{array} \\ \text { calcular un porcentaje global de cada clase. (Vasconcelos et al., 2020). } \\ \text { Red neuronal de una sola capa (Grist et al., 2020). } \\ \text { DFM } & \begin{array}{l}\text { Se puede conectar a capas de convolución para mejorar la tasa de reconocimiento y acelerar el proceso de } \\ \text { entrenamiento de las redes neuronales profundas (Huang et al., 2020). }\end{array}\end{array}$

Fuente: Autor

En la Tabla III. se expone las técnicas de IA recurrente en las propuestas estudiadas. Estas técnicas se emplean por medio de diferentes tipos de clasificadores algorítmicos que proceden a agrupar en categorías o clases los datos, en este caso imágenes clínicas, para la determinación por etapas de patrones, características lesiones o anormalidades que se escapan a la visión Humana o profesional de diagnóstico.

Al clasificar las lesiones o patologías presentes en cada grupo de imágenes según el objetivo médico a estudiar o partes específicas del cuerpo humano, se supera limitaciones a nivel de identificación de patrones de imagen, por métodos tradicionales de análisis de DI.

\section{Limitaciones y Alcances.}

En esta sesión se enuncia las limitaciones teniendo en cuenta que en cada artículo o proyecto estudiado se presentaron inconvenientes, algunos con soluciones dentro de los alcances y objetivos de cada estudio y otros como fundamento para tener en cuenta en posibles proyectos a futuro dentro de la misma área temática. 
Tabla IV. Limitaciones en los estudios de aplicación de técnicas de IA en DI

\begin{tabular}{|c|c|}
\hline Literatura & Limitaciones \\
\hline (Fos Guarinos, 2016) & $\begin{array}{l}\text { Conjunto de datos de imágenes insuficiente para poder extraer características determinantes o que } \\
\text { son alteraciones muy poco evidentes y difíciles de detectar. }\end{array}$ \\
\hline (Iturrioz et al., 2018) & Tiene margen de mejora y no debe tomarse como una herramienta diagnóstica funcional. \\
\hline (Lee et al., 2020) & Conjunto de datos desequilibrado. \\
\hline (Chen et al., 2020) & $\begin{array}{l}\text { La bronconeumonía y la bronquiolitis se clasifican erróneamente más fácilmente que otras } \\
\text { enfermedades. }\end{array}$ \\
\hline (Nguyen et al., 2020) & Se requiere costosos dispositivos de adquisición de datos y la cooperación de los pacientes. \\
\hline (Tandel et al., 2020) & Se limita a los datos de un solo instituto. \\
\hline (Battineni et al., 2020) & $\begin{array}{l}\text { La identificación de la EA en etapas muy tempranas es extremadamente difícil y no existen } \\
\text { herramientas para su detección simple. }\end{array}$ \\
\hline (Vasconcelos et al., 2020) & La clasificación errónea puede causar problemas graves al paciente. \\
\hline (Faleiros et al., 2020) & El estudio fue retrospectivo. Además, la cantidad de pacientes fue relativamente pequeña. \\
\hline (Grist et al., 2020) & $\begin{array}{l}\text { Bajo número de participantes en el grupo de ependimoma lo cual es un desafío común en los } \\
\text { estudios de imágenes pediátricas. }\end{array}$ \\
\hline
\end{tabular}

Fuente: Autor

Continuar desarrollando herramientas o algoritmos dentro del campo de la IA, que conviertan la información presentada en las imágenes clínicas en datos o resultados estructurados y clasificados para un mejor análisis y determinación de un diagnóstico. Esto se hace efectivo combinado con la habilidad del profesional dentro del campo de imágenes médicas. En Tabla $\mathbf{V}$ se presentó puntos de partida para continuar investigando y avanzando en la detección de diferentes afectaciones clínicas.

\section{Fundamentación Matemática.}

La función de entropía cruzada binaria, permite reducir el sesgo en los resultados durante la clasificación de las imágenes, está se enfoca en la clase durante el entrenamiento del método o algoritmo de IA. Los valores determinantes se pueden obtener en función del valor exacto o real de muestras o imágenes en cada clase dentro del grupo total de datos. La finalidad principal es reducir el sesgo en el clasificador o técnica de IA. La Tabla VI. Permite visualizar la estructura matemática de la función de entropía cruzada binaria y ponderada, una transformación para la proyección de una imagen diagnóstico por tecnología tradicional y una con automatización computacional.

Tabla VI. Fundamento matemático de técnicas de IA

\begin{tabular}{|c|c|}
\hline Fórmula matemática & Descripción de Formula. \\
\hline$X_{P}\left(x_{i}, y_{i}\right)=\frac{1}{N} \sum_{k=1}^{N} e^{\beta \frac{\max \left(X_{C T}\left(x_{i}, y_{i}, z_{k}\right)-1024\right)+1024}{1000}}$ & $\begin{array}{l}\text { Transformación para la proyección de unidades de TC. Donde } \\
X_{C T} \text { es la tomografía computarizada después de retirar la } \\
\text { mesa de TC y } X_{P} \text { es la radiografía simulada (Iturrioz et al., } \\
2018 \text { ). } \\
\text { pérdida de entropía cruzada para una red de clasificadores, } \\
\text { donde } Y^{\text {dec }} L^{\text {dec y C son el vector de salida del }} \\
\text { decodificador, el vector codificado en caliente y el número de } \\
\text { unidades ocultas, respectivamente (Lee et al., 2020). }\end{array}$ \\
\hline 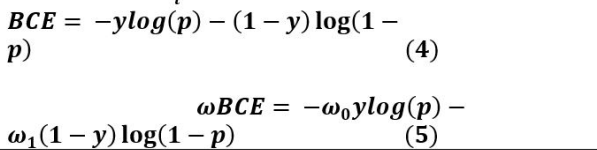 & $\begin{array}{l}\text { Versión modificada de la entropía cruzada binaria, } \\
\text { denominada entropía cruzada binaria ponderada }(\omega B C E) \text {, que } \\
\text { reduce el sesgo en el clasificador entrenado (Nguyen et al., } \\
2020) \text {. }\end{array}$ \\
\hline
\end{tabular}

Fuente: Autor 


\section{E. Técnicas de IA en rayos X y TC para la detección de COVID 19.}

En esta sesión se seleccionaron algunos proyectos sobre avances recientes en tres modalidades de imágenes diagnóstico para la detección temprana y eficaz del covid 19.

Tabla VII. Avances en el covid -19 con la implantación de técnicas de IA en imágenes diagnóstico.

\begin{tabular}{lcll}
\hline \multicolumn{1}{c}{ Literatura } & Modalidad & \multicolumn{1}{c}{ Método/Técnica } & \multicolumn{1}{c}{ Precisión } \\
\hline $\begin{array}{l}\text { (Apostolopoulos \& } \\
\text { 2020) }\end{array}$ & Mpesiana, & $\begin{array}{l}\text { Red neuronal profunda (DNN). } \\
\text { Transfer Learning. }\end{array}$ \\
(Bekhet et al., 2020) & RX & CNN. & $96,78 \%$ \\
& & & $96 \%$ \\
(Born et al., n.d.) & US & ResNet : POCOVID-Net & $98,0 \%$ \\
(Sotoudeh et al., 2020) & TC & ResNet-50. & $96,7 \%$ \\
(Elaziz et al.., 2020) & RX & FrMEM & $96,09 \%$ \\
\hline
\end{tabular}

Fuente: Autor

Como se muestra en la Tabla VII. Se lograron en los estudios presentes, precisiones por encima del $90 \%$ para la determinación de la enfermedad del covid 19. Las técnicas usadas a base de redes neuronales convolucionales permiten una mejor clasificación de las imágenes de tórax o pulmones para determinar la clase perteneciente de cada imagen de entrada, si pertenece o no a la enfermedad del covid 19, ó a otras enfermedades de tipo pulmonar.

Por ejemplo, la ecografía pulmonar no solo puede desempeñar un papel clave en el diagnóstico de enfermedades, sino que también puede utilizarse para supervisar la evolución de la enfermedad mediante controles periódicos realizados de forma no invasiva y sin necesidad de reubicación (Born et al., n.d.).

\section{Conclusión}

Actualmente se ha evidenciado un avance tecnológico, el cual no debe limitarse a simular funciones para el procesamiento manual de la información, sino que estas herramientas deben ser un soporte fundamental para el logro de la calidad de vida humana. La tecnología ha tomado un propósito importante, dependiendo de las formas como se realicen las tareas cotidianas. Estos avances contribuyen a una revolución tecnológica, lo que exige un cambio de paradigmas en el uso de procedimientos tradicionales e implementación de estas nuevas tecnologías dentro del área de la Medicina como es el caso del procedimiento de toma de imágenes diagnóstico y los beneficios de incluir herramientas de Inteligencia Artificial en esto.

Con este artículo se evidenció gran parte de los avances en tecnologías de IA para un diagnóstico temprano y seguro de diferentes patologías o enfermedades, desde imágenes localizadas en el cerebro, glándulas mamarias, músculos, sistema óseo, pulmones entre otras partes. De las Tecnologías más recurrentes como base de un nuevo método o reestructuración de éste mismo, se encuentra ResNet un algoritmo de red neuronal residual que ha permitido como método de clasificación la determinación del sesgo en la imagen clínica, esto con el fin de categorizar al grupo de imágenes o datos de entrada en una clase con los mayores detalles incluidos o mapeo de una imagen, GoogLeNet red neuronal estructurada codificador-decodificador y SVM algoritmos de aprendizaje supervisado en el proceso de clasificación.

Actualmente continúan los estudios y avances en esta área, y con este artículo se brinda una visión y punto de partida para posibles trabajos o proyectos a futuro. Gran parte de la información estudiada presentan ideas a abordar o posibles alcances para nuevos proyectos de investigación y aplicación. 
Entonces es pertinente continuar desarrollando proyectos precisos para el dictamen y gestión a tiempo de enfermedades o patologías como el cáncer de mama, neumonía, covid 19, etc.

\section{Referencias}

Apostolopoulos, I. D., \& Mpesiana, T. A. (2020). Covid-19: automatic detection from X-ray images utilizing transfer learning with convolutional neural networks. Physical and Engineering Sciences in Medicine, 43(2), 635-640. https:// doi.org/10.1007/s13246-020-00865-4

Battineni, G., Chintalapudi, N., Amenta, F., \& Traini, E. (2020). A Comprehensive Machine-Learning Model Applied to Magnetic Resonance Imaging (MRI) to Predict Alzheimer's Disease (AD) in Older Subjects. Journal of Clinical Medicine, 9(7), 2146. https://doi.org/10.3390/jcm9072146

Bekhet, S., Hassaballah, M., Kenk, M. A., \& Hameed, M. A. (2020). An Artificial Intelligence Based Technique for COVID-19 Diagnosis from Chest X-Ray. 2nd Novel Intelligent and Leading Emerging Sciences Conference, NILES 2020, 191-195. https://doi.org/10.1109/ NILES50944.2020.9257930

Born, J., Brändle, G., Cossio, M., Disdier, M., Goulet, J., \& Roulin, J. (n.d.). POCOVID-Net : A UTOMATIC D ETECTION OF COVID-19 $F$ ROM A N EW L UNG U LTRASOUND I MAGING D ATASET ( POCUS ).

Chen, K. C., Yu, H. R., Chen, W. S., Lin, W. C., Lee, Y. C., Chen, H. H., Jiang, J. H., Su, T. Y., Tsai, C. K., Tsai, T. A., Tsai, C. M., \& Lu, H. H. S. (2020). Diagnosis of common pulmonary diseases in children by X-ray images and deep learning. Scientific Reports, 10(1), 1-9. https:// doi.org/10.1038/s41598-020-73831-5

Elaziz, M. A., Hosny, K. M., Salah, A., Darwish, M. M., Lu, S., \& Sahlol, A. T. (2020). New machine learning method for imagebased diagnosis of COVID-19. PLoS ONE, 15(6). https://doi. org/10.1371/journal.pone.0235187

Expósito Gallardo, M. del C., \& Ávila Ávila, R. (2008). Aplicaciones de la inteligencia artificial en la Medicina: perspectivas y problemas. Acimed, 17(5), 0-0.

Faleiros, M. C., Nogueira-Barbosa, M. H., Dalto, V. F., Júnior, J. R. F., Tenório, A. P. M., LuppinoAssad, R., Louzada-Junior, P., Rangayyan, R. M., \& De Azevedo-Marques, P. M. (2020). Machine learning techniques for computer-aided classification of active inflammatory sacroiliitis in magnetic resonance imaging. Advances in Rheumatology, 60(1). https://doi.org/10.1186/ s42358-020-00126-8

Fos Guarinos, B. (2016). Diseño de técnicas de inteligencia artificial aplicadas a imágenes médicas de rayos $X$ para la detección de estructuras anatómicas de los pulmones $y$ sus alteraciones. https://riunet.upv.es:443/ handle/10251/70103

Grist, J. T., Withey, S., MacPherson, L., Oates, A., Powell, S., Novak, J., Abernethy, L., Pizer, B., Grundy, R., Bailey, S., Mitra, D., Arvanitis, T. N., Auer, D. P., Avula, S., \& Peet, A. C. (2020). Distinguishing between paediatric brain tumour types using multi-parametric magnetic resonance imaging and machine learning: A multi-site study. NeuroImage: Clinical, 25(December 2019), 102172. https://doi.org/10.1016/j. nicl.2020.102172

Huang, Z., Xu, H., Su, S., Wang, T., Luo, Y., Zhao, X., Liu, Y., Song, G., \& Zhao, Y. (2020). A computer-aided diagnosis system for brain magnetic resonance imaging images using a novel differential feature neural network. Computers in Biology and Medicine, 121(May), 103818. https://doi.org/10.1016/j. 
compbiomed.2020.103818

Iturrioz, M., Pascau, J., \& Estépar, R. S. J. (2018). EMPHYSEMA QUANTIFICATION ON SIMULATED X-RAYS THROUGH DEEP LEARNING TECHNIQUES Applied Chest Imaging Laboratory, Brigham and Women 's Hospital, Boston, MA, USA † Dept. de Bioingeniería e Ingeniería Aeroespacial , Universidad Carlos III de Madrid ,. Isbi, 273276.

Le, E. P. V., Wang, Y., Huang, Y., Hickman, S., \& Gilbert, F. J. (2019). Artificial intelligence in breast imaging. Clinical Radiology, 74(5), 357366. https://doi.org/10.1016/j.crad.2019.02.006

Lee, C., Jang, J., Lee, S., Kim, Y. S., Jo, H. J., \& Kim, Y. (2020). Classification of femur fracture in pelvic X-ray images using meta-learned deep neural network. Scientific Reports, 10(1), 1-12. https://doi.org/10.1038/s41598-020-70660-4

Nakata, N. (2019). Recent technical development of artificial intelligence for diagnostic medical imaging. Japanese Journal of Radiology, 37(2), 103-108. https://doi.org/10.1007/s11604-0180804-6

Nguyen, D. T., Kang, J. K., Pham, T. D., Batchuluun, G., \& Park, K. R. (2020). Ultrasound image-based diagnosis of malignant thyroid nodule using artificial intelligence. Sensors (Switzerland), 20(7). https://doi.org/10.3390/s20071822

Sarhan, A. M. (2020). Brain Tumor Classification in Magnetic Resonance Images Using Deep Learning and Wavelet Transform. Journal of Biomedical Science and Engineering, 13(06), 102-112. https://doi.org/10.4236/ jbise.2020.136010

Skandha, S. S., Gupta, S. K., Saba, L., Koppula, V. K., Johri, A. M., Khanna, N. N., Mavrogeni,
S., Laird, J. R., Pareek, G., Miner, M., Sfikakis, P. P., Protogerou, A., Misra, D. P., Agarwal, V., Sharma, A. M., Viswanathan, V., Rathore, V. S., Turk, M., Kolluri, R., ... Suri, J. S. (2020). 3-D optimized classification and characterization artificial intelligence paradigm for cardiovascular / stroke risk stratification using carotid ultrasound-based delineated plaque : Atheromatic TM 2 . 0. Computers in Biology and Medicine, 125(July), 103958. https://doi. org/10.1016/j.compbiomed.2020.103958

Sotoudeh, H., Tabatabaei, M., Tasorian, B., Tavakol, K., Sotoudeh, E., \& Moini, A. L. (2020). Artificial intelligence empowers radiologists to differentiate pneumonia induced by COVID-19 versus influenza viruses. Acta Informatica Medica, 28(3), 190-195. https:// doi.org/10.5455/aim.2020.28.190-195

Tandel, G. S., Balestrieri, A., Jujaray, T., Khanna, N. N., Saba, L., \& Suri, J. S. (2020). Multiclass magnetic resonance imaging brain tumor classification using artificial intelligence paradigm. Computers in Biology and Medicine, 122(March), 103804. https://doi.org/10.1016/j. compbiomed.2020.103804

Vasconcelos, F. F. X., Sarmento, R. M., Rebouças, P. P., Hugo, V., \& Albuquerque, C. De. (2020). Engineering Applications of Artificial Intelligence Artificial intelligence techniques empowered edge-cloud architecture for brain CT image analysis. Engineering Applications of Artificial Intelligence, 91(February), 103585. https://doi.org/10.1016/j.engappai.2020.103585

Wang, D., Xu, P. D. J., Zhang, M. S. Z., Li, M. S. S., Ph, D., Zhang, X., Zhou, P. D. Y., Zhang, X., Lu, Y., \& Ph, D. (2020). Evaluation of Rectal Cancer Circumferential. 1-9. https://doi.org/10.1097/ DCR.0000000000001519

Zhang, X., Lin, X., Zhang, Z., Sun, X., Sun, D., 
\& Yuan, K. (2020). Artificial Intelligence Medical Ultrasound Equipment: Application of Breast Lesions Detection. https://doi. org/10.1177/0161734620928453 